\title{
Estado mental de apego no resuelto, cuidado materno y seguridad del apego en dos diadas madre-hijo/a ${ }^{1}$
}

\section{Unresolved attachment state of mind, maternal care and child attachment security in two mother-child dyads}

\section{Estado mental de apego não resolvido, cuidado maternal e segurança do apego infantil em duas díades mãe-filho}

\author{
Magaly Nóblega ${ }^{1}$, ORCID 0000-0001-6572-813X \\ Laura Szteren ${ }^{2}$, ORCID 0000-0001-7682-6202 \\ Francesco Marinelli ${ }^{3}$, ORCID 0000-0002-0470-0130 \\ Patricia Bárrig-Jó ${ }^{4}$, ORCID 0000-0003-4685-5760 \\ Juan Núñez del Prado ${ }^{5}$, ORCID 0000-0001-5502-514X \\ Gabriela Conde ${ }^{6}$, ORCID 0000-0002-6103-1025

\section{1, 3, 4, 5, 6 Departamento de Psicología, Pontificia Universidad Católica del Perú \\ ${ }^{2}$ Uruguay Crece Contigo. Ministerio de Desarrollo Social de Uruguay}

Resumen: El presente estudio busca describir las características de las representaciones de apego de dos madres clasificadas con un estado mental de apego no resuelto, así como las características particulares de la interacción con su hijo/a: la calidad de su cuidado y la seguridad del apego del niño/a. Para las representaciones se empleó la Entrevista de Apego Adulto, para evaluar el cuidado de las madres el Maternal Behavior Q-Set 2.1, y la seguridad en el apego del niño/a se evaluó a través del Attachment Q-Set 3.0. Los resultados muestran que a pesar de que ambas madres tienen un apego no resuelto en función a experiencias de abuso vividas en la infancia, la sub-clasificación (apego Seguro en un caso e Inseguro en otro) está asociada a diferencias en la manera de percibir y responder a las necesidades de su hijo/a y en la seguridad del apego de éstos últimos.

Palabras clave: representaciones de apego adulto; relaciones de apego; cuidado infantil; abuso físico

Abstract: This study aims to describe the attachment representations of two mothers classified as unresolved in their state of mind with respect to attachment, as well as the specific characteristics of their interactions with their children: the quality of their care and the security of the child's attachment. The Adult Attachment Interview was used to evaluate attachment representations, the Maternal Behavior Q-Set 2.1 was used for the quality of maternal sensitivity, and the security of the child's attachment was evaluated with the Attachment Q-Set 3.0. The results show that while attachment is unresolved in both mothers due to their own experiences of childhood abuse, their subclassification (secure attachment in one case and insecure in the other) is associated with differences in how they perceive and respond to the child's needs, and in the security of the child's attachment. Keywords: adult attachment representations; attachment relations; children care; physical abuse

\section{(cc) $\mathrm{EY}$}

Esta obra está bajo una licencia de Creative Commons Reconocimiento 4.0 Internacional

\footnotetext{
${ }^{1}$ Este artículo de investigación se deriva de un estudio de mayor escala denominado "Canción de cuna como una de las estrategias de regulación emocional y sus relaciones con la sensitividad materna, las representaciones mentales maternas del apego y los precursores del apego infantil”, proyecto diseñado por Marina Altmann PhD., Equipo ATI -Atención y Desarrollo a la Temprana Infancia y a su Familia- y Olga Alicia Carbonell, PhD., Universidad Javeriana-Colombia; realizado por el Grupo de Investigación Relaciones Vinculares y Desarrollo Socioemocional y financiado por la Pontificia Universidad Católica del Perú con el código DGI-70242-3110. Realizado entre marzo del 2013 y junio del año 2014. Área Psicología, Sub-área Psicología.
} 
Resumo: O presente estudo busca descrever as características das representações de apego de duas mães classificadas com estado mental de apego não resolvido, bem como as características específicas de suas interações com seus filhos: a qualidade do cuidado e a segurança do apego da criança. O Adult Attachment Interview foi usado para avaliar as representações de apego, o Maternal Behavior Q-Set 2.1 foi usado para a qualidade da sensibilidade materna e a segurança do apego da criança foi avaliada com o Attachment Q-Set 3.0. Os resultados mostram que apesar do fato de ambas as mães possuírem um apego não resolvido baseado em experiências de abuso vividas na infância, sua subclassificação (apego seguro em um caso e inseguro no outro) está associada a diferenças em como elas percebem e respondem às necessidades da criança, e na segurança do apego da criança.

Palavras-chave: representações de apego de adultos, relações de apego, cuidado de crianças, abuso físico

Cómo citar:

Nóblega, M., Szteren, L., Marinelli, F., Bárrig-Jó, P., Núñez del Prado, J., \& Conde, G. (2020). Estado mental de apego no resuelto, cuidado materno y seguridad del apego en dos diadas madre-hijo/a. Ciencias Psicológicas, 14(2), e-2315. doi: https://doi.org/10.22235/cp.v14i2.2315

Correspondencia: Magaly Nóblega; e-mial: mnoblega@pucp.pe. Laura Szteren; e-mail: szteren@gmail.com. Francesco Marinelli; e-mail: fmarinelli@pucp.pe.Patricia Bárrig-Jó; e-mail: pbarrig@pucp.pe. Juan Núñez del Prado; e-mail: j.nunezdelprado@pucp.pe.Gabriela Conde; e-mail: gabriela.conde@pucp.pe

El estudio del apego en la adultez sostiene que las personas tienen un repertorio de representaciones de las experiencias tempranas con sus cuidadores, lo que Main, Kaplan y Cassidy (1985) denominaron estados mentales con relación al apego. Estos estados mentales, funcionan como una serie de reglas conscientes y/o inconscientes que organizan la percepción de la información relativa a las experiencias de apego en el adulto (Bowlby, 1969/1982; Main et al., 1985), teniendo una implicancia directa en las conductas parentales. Así, en diversos estudios se ha encontrado una relación directa entre los estados mentales de apego de la madre y la conducta sensible de cuidado hacia su hijo o hija (Behrens, Haltigan, \& Bahm, 2016; Madigan et al., 2006; van IJzendoorn, 1995; Verhage et al., 2016).

Los estados mentales en relación al apego se reflejan en la manera en que la persona articula y organiza su discurso para dar cuenta de las experiencias con sus cuidadores significativos y también en las estrategias que emplea en el proceso. Así, en base a la clasificación realizada por George, Kaplan y Main (1985), las personas pueden presentar un patrón de apego organizado, que corresponde a las categorías de Seguro-Autónomo, InseguroPreocupado e Inseguro-Desentendido; o un patrón desorganizado denominado: No resuelto/Desorganizado. Esta última categoría se asigna ante la presencia de signos de desorientación y desorganización en el estado mental frente a situaciones de pérdida o violencia (Main \& Hesse, 1990). Sin embargo, Hesse (2016) señala que debido a que estas manifestaciones se presentan frente a experiencias específicas y pueden ser temporales, a la clasificación de apego no resuelto siempre se le asigna una sub-clasificación alterna correspondiente a un patrón organizado. En ese sentido, más allá de que una persona tenga un 
patrón No resuelto ante ciertas experiencias, tiene un modo de organización general que responde a un apego organizado.

Las experiencias de pérdida o violencia representan potenciales eventos traumáticos ante las cuales se puede configurar un estado mental de apego no resuelto. Esto se debe a que en los casos en los que ha habido una pérdida del progenitor, la ausencia de éste incrementa el riesgo físico y la falta de la sensación de bienestar y seguridad que generaba dicha relación (Bowlby, 1969/1982; Fraley \& Shaver, 2016). Por otro lado, cuando se encuentran experiencias de abuso (físico o sexual), las fuentes de seguridad del infante se convierten simultáneamente en fuente de peligro por lo que la persona se enfrenta a un trauma de naturaleza crónica (Main \& Hesse, 1990). Berthelot et al. (2015) comprobaron que las personas con historias de pérdida o abuso durante su infancia tienen mayor probabilidad de desarrollarlo, encontrando en su estudio que el $42 \%$ de madres con historias de abuso o negligencia presentaban un apego no resuelto. Frente a esto, Hesse (2016) plantea que, si en la adultez estas experiencias se mantienen poco integradas, evidenciando una falta de conciencia clara de lo ocurrido y de sus implicancias, se suelen presentar signos de desorientación y desorganización frente a dichos eventos traumáticos o situaciones posteriores que remitan a los mismos, como puede ocurrir en la crianza de los hijos. Por eso, el estudio de la clasificación de apego No resuelto ha adquirido gran importancia al considerarse un factor de riesgo en el ejercicio de la maternidad y el cuidado infantil (LyonsRuth \& Jacobvitz, 1999).

Adicionalmente, el estudio del apego no resuelto se hace especialmente relevante en ciertos contextos donde su incidencia aumenta. Así, Bakermans-Kranenburg y van IJzendoorn (2009) encontraron que esta clasificación de apego se encuentra presente en un $32 \%$ en poblaciones de alto riesgo, como madres de estratos socioeconómicos bajos o en un $38 \%$ en contextos de violencia familiar. Todos estos elementos cobran especial relevancia en contextos como el peruano en el que entre el 19 y $21 \%$ de los menores de 14 años no viven con ambos padres, el $58.8 \%$ de las mujeres reportan haber recibido castigo físico por parte de sus propios padres durante la infancia y especialmente el 34\% de las madres con niñas/os de 1 a 5 años de edad declararon que experimentaron alguna forma de violencia física y/o sexual (Instituto Nacional de Estadística e Informática, 2016). Adicionalmente a esto, el 29.6\% de los hogares de Lima Metropolitana pertenecen a los sectores socioeconómicos D y E (APEIM, 2017).

Profundizar en las representaciones de apego de las madres adquiere importancia debido a que diversos estudios sostienen que éstas se encuentran asociadas a la sensibilidad que despliegan en el cuidado hacia sus hijos/as (Behrens et al., 2016; Madigan et al., 2006; van IJzendoorn, 1995; Verhage et al., 2016). Así, los metaanálisis indican que los progenitores con apego no resuelto son más propensos a desarrollar relaciones desorganizadas con sus hijos/as (van IJzendoorn, 1995; Verhage et al., 2016). Hesse y Main (2000) consideran que las experiencias traumáticas no resueltas pueden generar la intrusión de recuerdos aterradores parcialmente disociados durante la interacción con el infante. Estos aspectos del propio mundo afectivo traumático y no integrado del cuidador son reactivados ante las manifestaciones afectivas y las iniciativas de apego del infante (Hsiao, Koren-Karie, Bailey, \& Moran, 2015; Lyons-Ruth \& Jacobvitz, 1999) lo cual daría lugar a respuestas impredecibles, disruptivas o inadecuadas (Madigan et al., 2006; van IJzendoorn \& Bakermans-Kranenburg, 1996).

De esta manera, estas madres presentan señales insuficientes para monitorear su comportamiento en las interacciones con el niño (Schuengel, Bakermans-Kranenburg, \& van IJzendoorn, 1999). Hsiao et al. (2015) encontraron que madres con apego no resuelto presentan ciertas dificultades en su habilidad para guiar y sostener de manera efectiva las interacciones con sus hijos e hijas, así como para involucrar de manera efectiva al niño/a en actividades compartidas. Sostienen que esto se debe a que la madre adopta un rol pasivo, donde es ella quien se queja o lamenta por la atención que le brinda su hijo/a. Del mismo modo, Crugnola et al. (2013) encuentran en díadas de madres e hijos/as menores a un año que este estado de la mente 
no resuelto se asocia significativamente $(r=.33)$ con un involucramiento negativo de la madre hacia el niño/a y con un menor involucramiento durante actividades de juego $(r=-.45)$. Por otro lado, en las madres con antecedentes de abuso, lo que con frecuencia suele explicar o estar a la base de la clasificación de apego no resuelto, Moehler, Biringen y Poustka (2007) encontraron menores niveles de sensibilidad, no-intrusividad y no-hostilidad en comparación al grupo control, enfatizando que el comportamiento intrusivo sería la característica más resaltante en estas madres ligada al apego desorganizado, denotando así una falta de respuesta adecuada y menores niveles de disponibilidad emocional ante las necesidades de sus hijos.

En este contexto de interacciones difíciles con sus hijos, las madres pueden llegar a mostrar un comportamiento hostil (Lyons-Ruth \& Jacobvitz, 1999). Frente a esto, Reijman et al. (2016) encontraron que el $42 \%$ de las madres que ejercían maltrato hacia sus hijos/as presentaban un apego no resuelto, mientras que en el grupo de madres que no lo hacían, esta clasificación solo se encontraba presente en un 17\%. Del mismo modo, Bakermans-Kranenburg y van IJzendoorn (2009) encontraron que entre aquellas personas que ejercen violencia al interior del ámbito familiar (por ej. hacia su hijo/a), el 38\% presentaban este tipo de apego.

A pesar de lo señalado, ciertos estudios muestran que el efecto negativo del apego no resuelto en las conductas de cuidado puede variar en función al patrón de apego organizado que la persona presente como sub-clasificación alterna (Seguro, inseguro-preocupado o insegurodesentendido). Según Ballen, Demers y Bernier (2006), puede esperarse que los adultos con una clasificación no resuelto/inseguro tengan mayores dificultades en la interacción y crianza de los hijos. Estudios muestran que madres con esta clasificación de apego manifiestan una mayor conducta atemorizada/atemorizante en presencia de su bebé y un aumento de los comportamientos intrusivos (Goldberg, Benoit, Blokland, \& Madigan, 2003; Schuengel et al., 1999). Por otro lado, aquellas con un estado de la mente no resuelto/seguro demuestran una mayor cercanía en las interacciones con sus hijos y comportamientos menos atemorizantes comparadas con las primeras (Ballen et al., 2006; Saunders, Jacobvitz, Zaccagnino, Beverung, \& Hazen, 2011).

Por otro lado, los estudios realizados han confirmado una importante correspondencia entre el estado de la mente del adulto cuidador, su comportamiento sensible y el patrón de apego del infante a su cargo (Ainsworth, Blehar, Waters, \& Wall, 1978; Behrens et al., 2016; Madigan, Hawkins, Plamondon, Moran, \& Benoit, 2015; Verhage et al., 2016). En el caso de los niños cuyas madres presentan un estado de la mente no resuelto, su sistema de apego se activará al experimentar temor frente los comportamientos de la madre, motivándolo a buscar la protección de ésta; sin embargo, debido a que esta figura es la fuente de alarma, el niño entra en un dilema irresoluble en el que no puede realizar ninguna estrategia coherente; esta situación lleva al niño a un comportamiento desorganizado (Main \& Hesse, 1990). En el metaanálisis más reciente realizado por Verhage y colaboradores (2016) se encontró que el $42 \%$ de madres que presentaban un apego no resuelto tenían a su vez niños/as con apego desorganizado. Asimismo, Berthelot et al. (2015) encontraron que el apego no resuelto de la madre correspondía en un $92 \%$ con un apego inseguro del hijo/a, y un 67\% con conductas de apego desorganizadas del mismo. En ese sentido, es posible esperar que los niños de madres con apego no resuelto tengan dificultades para construir una imagen segura de sus figuras de apego y de su entorno lo cual dificulta el establecimiento de un vínculo de apego seguro.

Considerando lo expuesto, el presente estudio busca describir las representaciones de apego de dos madres clasificadas con apego no resuelto y las características que presentan en la calidad de su conducta materna (sensibilidad) y la seguridad del apego de su hijo o hija. 


\section{Método}

\section{Diseño}

El presente estudio forma parte de una investigación multicéntrica diseñada por Altmann, Equipo ATI y Carbonell con el objetivo de explorar el aporte de la sensibilidad materna, las estrategias regulatorias de la madre y sus representaciones de apego sobre la seguridad del apego del niño durante el primer año de vida (Bárrig-Jó et al., 2016; Nóblega et al., 2016). De forma específica, en el presente artículo se plantea el estudio de dos casos, los cuales fueron seleccionados por presentar características comunes relevantes para el propósito de la investigación. Ambos casos corresponden a madres que presentan una clasificación de apego no resuelto, pertenecen a un nivel socioeconómico bajo y reportan historias de violencia durante la niñez. Así, se busca describir cualitativamente el discurso de estas dos madres a partir de la entrevista de apego adulto (AAI) así como las conductas más características del vínculo entre ellas y su hijo o hija observadas en un momento de interacción libre.

\section{Participantes}

La primera participante, identificada con el seudónimo de "Luisa", tenía 33 años y educación secundaria completa. En el momento de la entrevista estaba casada y se dedicaba a las labores del hogar. Luisa vivía en una casa con su familia y junto a sus suegros. Ella tenía 3 hijos de 15 y 6 años y la última de 9 meses con quien participó en el estudio. Como antecedentes importantes, la niña estuvo hospitalizada a raíz de una enfermedad; adicionalmente, la madre se separó de su hija por menos de una semana debido a una emergencia familiar, quedando la niña a cargo de su hermana mayor.

La segunda participante, identificada con el seudónimo de "Claudia", tenía 23 años y estudios secundarios completos. En el momento de la entrevista, era conviviente y se dedicaba a las labores del hogar. Su único hijo de 9 meses fue con quien participó en el estudio. Claudia también se separó de su hijo por un lapso menor a una semana como consecuencia de la enfermedad de un familiar; durante esta separación, el niño se quedó a cargo de su abuela.

Las madres aceptaron participar voluntariamente del estudio y de la entrevista del AAI firmando un consentimiento informado donde además se garantizó el anonimato de las participantes. Inicialmente fueron entrevistadas un total de cinco madres; de ellas se seleccionaron a las dos madres descritas debido a que ambas fueron calificadas con un estado de la mente con respecto al apego no resuelto. Al finalizar el estudio, las cinco madres fueron invitadas a una última reunión para tener una entrevista y recibir orientación acerca de la crianza de su hijo(a).

\section{Medición}

Apego adulto. Para evaluar las representaciones de apego de las madres, se empleó la AAI (George, Kaplan, \& Main, 1985) en su versión en español (Main \& Goldwyn, 2002/2004). Se trata de un protocolo semi-estructurado de 18 preguntas que se enfocan en la exploración del estado mental de apego de una persona como reflejo de sus experiencias tempranas y en los efectos de éstas en su funcionamiento actual (George et al., 1985; Hesse, 2016). Considerando el contenido y la forma en la cual responde a la entrevista, se asigna una clasificación o categoría global: Seguro-Autónomo, Inseguro-preocupado, Inseguro-desentendido, No resuelto/Desorganizado o No se puede clasificar. En el caso de las dos últimas clasificaciones, se les debe asignar también la sub-clasificación alterna de alguno de los patrones organizados según mejor corresponda, ya sea Seguro o Inseguro (George et al., 1985).

El análisis de la entrevista incluye por un lado las valoraciones respecto a la conducta parental durante la infancia a partir del contenido de las experiencias referidas por las entrevistadas; y por otro lado incluye un análisis de la forma del discurso lo cual refiere a la 
coherencia del pensamiento respecto a dichas relaciones tempranas. Con respecto al análisis formal, se analiza tomando en cuenta las máximas de Grice (1975), las cuales son: la calidad, que se refiere a la certeza y evidencias de lo que se dice; la cantidad, es decir, la capacidad de ser sucinto y a la vez tener una respuesta completa; la relación, que implica brindar información relevante; la forma, que alude a proporcionar respuestas claras y ordenadas.

Adicionalmente, el apego no resuelto se asigna ante la presencia de signos de desorientación y desorganización frente a los eventos traumáticos vividos por la persona. Esto se puede manifestar en caídas en el monitoreo del pensamiento, del discurso o de la conducta durante la discusión de estos eventos, o cuando las experiencias se perciben poco integradas, confusas o evidenciándose una falta de conciencia clara de lo ocurrido y de sus implicancias (Hesse, 2016; Main \& Hesse, 1990).

Cabe señalar que a pesar de que el análisis se realiza sobre el contenido formal y la forma lingüística de las respuestas, las clasificaciones de la AAI han demostrado no estar relacionadas con la mayoría de las medidas de inteligencia, ni con la memoria a corto y largo plazo de experiencias no vinculadas al apego (Bakermans-Kranenburg \& van IJzendoorn, 1993; Hesse, 2016).

La administración de la entrevista dura una hora aproximadamente y se transcribe literalmente. La codificadora de ambas entrevistas fue una persona independiente al equipo de investigadores, ha sido entrenada y certificada por Main y Hesse, University of California Berkeley y trabajó exclusivamente con las transcripciones del discurso.

Sensibilidad materna. Para evaluar el comportamiento sensible de la madre, se empleó el Maternal Behavior Q-Set 2.1 (MBQS 2.1; Pederson \& Moran, 1995), en su versión en español elaborada por Posada y colaboradores (2002). Este instrumento evalúa el nivel de sensibilidad del cuidador en contextos cotidianos a través de 90 enunciados que describen un amplio rango de características generales de la calidad de la conducta del cuidador. Estas conductas incluyen aspectos como la sensibilidad en las respuestas de la madre a las señales del niño, el tono afectivo y la participación activa en las interacciones, la disponibilidad para tener en cuenta al niño aun cuando realiza otras actividades, entre otras.

La aplicación de este instrumento consiste en observar la interacción de la madre con su hijo(a) y distribuir los 90 enunciados en 9 grupos con 10 ítems cada uno que van desde los más característico a los menos característicos del comportamiento de la madre. Para obtener la puntuación de sensibilidad, los puntajes de los 90 ítems son correlacionados con los puntajes que describen a un cuidador altamente sensible (Pederson \& Moran, 1995).

Para el presente estudio se registró en video la interacción de la madre con su hijo o hija y ésta fue calificada por dos jueces independientes capacitados en el tema de sensibilidad. Ambos calificadores desconocían el tipo de apego de la madre. Las calificaciones presentaron una confiabilidad inter-evaluador de .865 y .719 para la diada 1 y 2 respectivamente calculada a partir del método de coeficiente intraclase.

Seguridad del apego. La conducta de apego del niño(a) se evaluó mediante el Attachment Q-Set 3.0 (AQS; Waters, 1995). Este instrumento evalúa la seguridad del apego de los niños a partir de la observación de la relación con su cuidador. La conducta se califica a través de 90 ítems referentes al comportamiento del niño/a, que incluyen la calidez en las interacciones con la madre, la búsqueda de proximidad y el disfrute del contacto físico con ella, las conductas de exploración, entre otros. Así, los ítems son organizados en 9 grupos de 10 ítems cada uno que van desde los comportamientos más característico a los menos característicos. Posteriormente, se obtiene la puntación global de la conducta de base segura, al comparar las puntuaciones obtenidas en los 90 ítems con los puntajes que describen un niño idealmente seguro (Waters, 1995).

Para el presente estudio se registró en video la interacción de la madre con su hijo en la casa y ésta fue calificada por dos jueces independientes capacitados en el instrumento y con 
conocimiento en el tema de apego. Ambos calificadores desconocían el tipo de apego de la madre. Las calificaciones de las dos díadas participantes del estudio presentaron una confiabilidad inter-evaluador de .912 y .721 respectivamente calculada a partir del coeficiente intraclase.

\section{Procedimiento}

La administración de los instrumentos se llevó a cabo como parte del conjunto de evaluaciones realizadas en la investigación multicéntrica previamente mencionada de la cual se desprende el presente estudio. En ese sentido, para este artículo se ha utilizado la información de la interacción libre entre madre e hijo(a) llevada a cabo en la primera reunión y de la AAI administrada en la cuarta reunión. Las observaciones de la interacción madre-hijo(a) se realizaron en las casas de las participantes, a las cuales asistió un observador para registrar en video las conductas en su ambiente natural. Por otro lado, las entrevistas se realizaron en el laboratorio de la universidad que financió el estudio, el cual era un espacio reservado donde solo se encontraban la participante y la entrevistadora.

\section{Resultados y Discusión}

Para la presentación de los resultados, se analizará en primer lugar el caso de Luisa y posteriormente a Claudia de manera independiente. Para ello, se tomarán algunos aspectos puntuales de las entrevistas que permiten describir y caracterizar el estado mental con relación al apego de cada una. Además, en ambos casos se describirán las conductas de sensibilidad materna y los comportamientos de seguridad en el apego de su hijo(a) que resultaron más característicos a partir de la observación realizada y que es posible explicar su presencia a partir del estado mental de apego asignado en cada caso.

\section{Luisa "danza entre ángeles y fantasmas"}

Cuando Luisa describe la relación con su madre en la infancia, menciona que ésta era "intolerante", "nerviosa", "muy ama de casa", "preocupada" y "atenta". Al preguntarle por qué la describía como intolerante, ella describe: "Sí, porque no era de esas mamás que entendía bien las cosas, nunca me preguntaba por qué ha pasado eso, por qué ha pasado el otro... su solución siempre era castigar". Así, Luisa describe diversos episodios de violencia por parte de la madre, como golpes con un palo, y también actitudes atemorizantes de la madre como asustarla con aquello que a ella le daba miedo, por ejemplo, la cabeza de un carnero.

De esta manera, la madre, principal figura de apego, es recordada como fuente de temor, lo cual implica para Luisa una paradoja irresoluble. Al hablar de esta temática, su discurso se vuelve desorientado, por momentos no puede nombrar el abuso o no puede terminar las frases, utiliza palabras sin sentido para nombrar el abuso: "Eeh yo le... digamos, pero mamá espera, noo pero mee, me empezaba a gritar y ta ta ta ta...mi mamá veía el pretexto y me pegaba con cualquier cosa porque era la engreída de mi papá de otra manera."

Además, se infiere por la narrativa, que su madre tuvo actitudes de rechazo del acercamiento de Luisa, ya que no era una figura a la cual ella pudiese acudir ante situaciones de estrés. Así, cuando de niña se sentía agobiada conversaba con su hermana, cuando se lastimaba tampoco recurría a su madre y cuando se enfermaba, su padre era quien la llevaba a los médicos. Actualmente, parece tampoco confiar en dicha disponibilidad, ya que, ante una separación por una situación de emergencia, dejó a su hija al cuidado de una hermana mayor de 15 años, esto mostraría que Luisa no confió en su propia madre para que cuide a su hija.

Cuando ejemplifica por qué describe a su madre como "nerviosa", relata:

"Sí, mire un montón de recuerdos, mi mamá es la que, por ejemplo, yo cogía una taza de agua o una taza de leche y inoo queee noo, te vas a caer, te vas!, o sea mi mamá es, ya veía que 
la taza de leche estaba derramada, que yo me había quemado, ya me habían llevado al hospital. O sea, antes de que pasen las cosas, ya lo, ella ya lo predisponía, pero no pensaba el lado bueno, ella siempre, el lado trágico o algo va a pasar, ¡no que mier!, pero si todavía no ha pasado nada pero jva a pasar! Ella, no, no, algunas, en algunos nivel llega a asustar y hasta ahora, por ejemplo, mi bebita está caminando jaaaaaiiihhh! No grite le digo porque idéjela! Si se cae que se levante. No, no sigo la ruta, sí, pero mi mamá es así, hasta ahora.

En este pasaje se observa que en su discurso hay cierta violación de la forma de la narrativa (según la clasificación de Grice, 1975) cuando utiliza un lenguaje exagerado ("un montón" o "siempre el lado trágico") o introduce diálogos que sostuvo con su madre como si estuviesen sucediendo en el presente. Así, se observa una caída en el monitoreo temporal de su estado mental frente a dichas experiencias, ya que pasa de estar hablando del pasado (su infancia) al presente $\mathrm{y}$ a hechos ocurridos con su propia hija. Esta forma de relatar las experiencias vividas con relación a su madre denota que sigue "envuelta" en la situación.

Luisa describe la relación actual con su madre: "Mi mamá me ayuda ahorita bastante, pero es muy metida [...] la veo como que quisiera enmendar algunas cosas. Mi mamá en varias oportunidades me ha pedido perdón por el maltrato". Luisa narra que actualmente hay aspectos de su madre que le siguen molestando, pero también elabora una posible explicación de la conducta de la madre ("la veo como que quisiera enmendar algunas cosas") y parece haber un perdón implícito hacia ella.

Por otro lado, cuando Luisa se refiere a la relación con el padre, los adjetivos que escoge para describirlo ("renegón", "detallista", "alegre", "compañero" y "responsable") no son ni muy positivos ni muy negativos. Con respecto al adjetivo "renegón”, expresa: "[...] cuando se exasperaba llegaba a gritar, nunca pegaba [...] No nos acordamos de episodios suyos fuertes, pero sí sabía que renegaba o se preocupaba por un trabajo, él renegaba o gritaba con mi mamá". Si bien también es descrito como un padre que gritaba y discutía mucho con la madre, refiere que el padre la defendía ante la madre ("estaba mi papá que me defendía"), que la hacía sentir muy especial. En general, estos adjetivos dados al padre se encuentran sustentados en su discurso, a partir de lo cual, se infiere que éste ha tenido actitudes de cuidado hacia ella. Tampoco se presentan violaciones significativas a las máximas de Grice. Afirma que tenía una relación más cercana con el padre que con la madre, y en su discurso ilustra que tener al padre como una fuente de soporte en su infancia ha sido importante para ella.

Además del padre y la madre, Luisa ha tenido otra figura importante en su vida, su madrina (la tía), quien también ofició como figura de sostén. "Ella me llevaba, en las vacaciones, y me llevaba a su casa donde era al revés de mi casa en el aspecto que mi mamá por su pro..., mi mamá no dejaba, ya no dejaba que jugáramos. [...] Y yo esperaba que llegara el fin de semana y otra vez que me.., intentar que [mi madrina] me lleve". Relata de este modo episodios de mayor tranquilidad con su madrina dada la ausencia de la violencia psicológica de la madre. Se observa además que la ira al hablar de su madre está contenida, por ejemplo, al no terminar la palabra "mi mamá por su pro...", quizás quería decir "por su problema" pero se corta y no se permite expresarlo de esa manera.

El discurso de Luisa es fresco y espontáneo, no estereotipado, con expresiones como "mira ahorita se me acaba de venir algo a la mente". Se observa un discurso coherente, la ira se muestra contenida y por momentos tiene un tono humorístico. Refiere estar involucrada en la relación con la madre porque presenta quejas activas hacia ella de las cuales Luisa es consciente; sin embargo, también hay un reconocimiento y una aceptación de cómo es su madre y su relación con ella, evidenciándose el perdón hacia las fallas que hayan podido tener, pero al mismo tiempo no se identifica con los aspectos negativos de ésta. Todas éstas son características de los estados de la mente con respecto al apego de tipo seguro.

Además, en su discurso se observa la presencia de procesos metacognitivos que tienen que ver con la posibilidad de monitorear y reportar el proceso de pensamiento durante el 
transcurso de la entrevista, lo cual es un muy buen indicador, que se presenta en apegos seguros. Específicamente estos procesos se refieren al reconocimiento de un cambio en la representación de la figura materna. Expresa: "Entonces, eso, era la parte que mi mamá, bueno yo después ya cuando era adolescente yo llegué a entender porque lo hacía, ¿no? [...] uno como niño no sabe los problemas que pasaron como pareja ¿no? Una manera de desahogar era, pero eso yo ya lo entendi después".

A partir de la clasificación y análisis de la entrevista, se llega a la conclusión que Luisa presenta una tendencia a un estado de la mente con respecto al apego no resuelto debido a experiencias de trauma de su infancia, pero a excepción de esas experiencias presenta características que permiten asignarle una sub-clasificación alterna de un apego seguroautónomo.

Fonagy, Steele, Moran, Steele y Higgit, (1993) sostienen que el AAI permite medir los "Fantasmas en la Habitación", metáfora que describe la forma en que las experiencias no resueltas impregnan la relación de una madre con su bebé. En este caso, las experiencias de Luisa de haber sufrido violencia se hacen presentes como fantasmas en la relación con sus propios hijos, al punto que le resulta muy difícil separarse de los mismos y que sean cuidados por otra persona que no sea ella, ya que dice que no confía en su madre para que cuide a su bebé. No obstante, pese a que presenta una no-resolución de las experiencias de violencia, presenta una subclasificación segura. En este caso, podría pensarse que la madrina de Luisa oficiaba como figura protectora alterna o "Ángel en la Habitación” (Lieberman, Padrón, Van Horn, \&Harris, 2005). Al respecto, diversos estudios rescatan la importancia de la presencia de figuras de cuidado alternas frente a experiencias negativas con los progenitores en la niñez; los autores sostienen que si éstas han brindado un adecuado soporte emocional facilitarán que la persona gane seguridad y pueda interiorizar otros patrones de relaciones conducentes a un estado de la mente más seguro (Saunders et al., 2011; Zaccagnino, Cussino, Saunders, Jacobvitz, \& Veglia, 2014). Así, entre estos "fantasmas" y "ángeles" Luisa convive y navega día a día, aunque por momentos unos se destaquen más que otros.

En relación a su comportamiento materno, Luisa muestra una buena habilidad para detectar, interpretar y responder a las necesidades de su hija $(r=.749)$. Principalmente, entre sus conductas más características se observa una respuesta sensible ante las señales de la niña, manteniendo un estado de ánimo positivo (MBQS, ítem 36) y un alto grado de participación en sus interacciones. Así, se presenta como algo característico en ella el poder darse cuenta de las señales de su hija (MBQS, ítem 1) y buscar interacciones cara a cara (MBQS, ítem 34). Resulta importante señalar que, si bien los sentimientos negativos hacia su hija pueden estar presentes, Luisa es capaz de dejarlos de lado cuando interactúa con ella (MBQS, ítem 22). De esta manera, y si bien Luisa presenta un apego no resuelto, su capacidad para reconocer los propios estados mentales y los de su hija, le permitiría mantener interacciones más reguladas y positivas con esta (Berthelot et al., 2015; Madigan et al., 2015).

Por otro lado, su hija ha logrado establecer un vínculo de seguridad con ella $(r=.383)$; en ese sentido, muestra calidez en las interacciones con ella y manifiesta apertura a explorar el entorno e interactuar con otras personas. Así, se puede observar a una niña alegre, juguetona (AQS, ítem 9) y activa la mayor parte del tiempo (AQS, ítem 68), siendo expresiva en sus interacciones (AQS, ítem 89) y sintiéndose atraída por actividades nuevas (AQS, ítem 85). Además, es una niña que no se enoja fácilmente, ni se muestra impaciente, exigente o molesta hacia los comportamientos de la madre (AQS, ítems 30, 38 y 65). Así, como señalan Hsiao et al. (2015), las madres con elementos de apego seguro brindarían mayores oportunidades a sus hijos para expresar emociones y necesidades para así poder elaborarlas. 
Claudia, "bucea" en medio de sus experiencias traumáticas infantiles

Claudia se crió con su madre, su padre y tres hermanos. Las experiencias de haber sufrido violencia física por parte de su padre y ser testigo de violencia doméstica, la marcaron en forma traumática y la narrativa de la entrevista se encuentra inundada por dichos relatos.

“...porque mi papá no era o sea no era la persona que cogía una correa ¿no?, o sea con lo que tenía él te daba, entonces este yo a eso temía, a que mi papá tenga algo juaa, me tira porque si mal no recuerdo una vez mi papá me..., [...] mi papa me jaló de mi cabello y me dejó, me tiró hacia el suelo, después, yo tengo una marca por aquí o por acá, algo así de mi cara (ajá) por qué, porque me tiró y.. y... yo caí de cara, asi me arañé yo, eso lo era lo que tengo más fuerte ¿no?" Otro episodio que relata es el siguiente: “...se me hinchó mi vista por porque por darme con la correa, la hebilla me chocó y me hizo un boliche."

Con respecto a la madre, dice que no le prestaba mucha atención: "Ah mi mamá cuando yo le decía mamá, este cómo se llama, ayúdame en mis tareas, mi mamá me decía 'no, no ves que tengo que vender, que date cuenta, que sí no vendo no voy a tener para darte de comer', algo asi". Así, el discurso de Claudia presenta altos niveles de rechazo y negligencia con respecto a las experiencias inferidas con sus progenitores.

Claudia no podía recurrir a sus padres ante situaciones de estrés, cuando se le pregunta acerca de un episodio en el que se haya sentido agobiada de niña, relata lo siguiente: "Lloraba, lloraba, lloraba...cuando se peleaban mis papás, a veces sentía que, decía, yo Dios mío me quiero morir. Este, a veces me quería salir de mi casa (...)”. Se observa que el tema de la violencia vuelve a surgir siendo una temática recurrente en su narrativa. Además, se destaca el hecho de que los episodios de violencia le han generado sentimientos depresivos e ideas de muerte.

Cuando se lastimaba físicamente, relata que buscaba la atención de su madre incluso infringiéndose más daño físico: "Trataba a veces que me salga, que me, que se me, que me haga mal la llaga, a veces cuando a veces me caía así, me repelaba para que se me haga más, pa pa que me pongan atención y así quizá ¿no? mi mamá me ponía atención, me curaba, y a veces, ahí yo le preguntaba tú me quieres?, decía mi mamá sí yo te quiero mucho, yo le decía no, tú no me quieres, y hasta ahora a veces."

A lo largo de la entrevista se observa que Claudia se encuentra confundida, temerosa y afligida por experiencias traumáticas. Esta temática aparece en la entrevista como predominante y además de forma incoherente, predomina el temor en varios fragmentos de la misma, dando la impresión que no puede alejarse de dicha temática y no lo puede controlar, ya que domina su proceso de pensamiento. Por ejemplo, en un pasaje de la entrevista cuando está hablando acerca de los adjetivos con respecto a la relación con el padre, afirma: "Me daba miedo de.. a veces así de verme me me me temblaba, me me daba cosa de verlos así, mejor yo me iba en donde mi abuela y le contaba a mi abuela así. [...] cuando veía a mi papá yo ya estaba temblando o si veía yo me quedaba calladita, serenita pa' que no, porque a él todo lo molestaba".

Además del contenido recurrente de violencia, en el discurso de Claudia hay violaciones a la máxima de colaboración de cantidad (Grice, 1975), es decir que da más información de la necesaria, ya que los turnos de conversación son largos en varios pasajes de la entrevista. Asimismo, existe evidencia de caídas en el monitoreo del discurso con respecto a las situaciones de trauma. Así, se observa que hay una desregulación afectiva y cognitiva cuando habla de las situaciones de violencia que sufrió, titubea y habla del miedo, lo cual genera un patrón de comportamiento maternal atemorizada-atemorizante.

En suma, Claudia sufrió violencia física con respecto al padre y fue testigo de violencia entre sus progenitores. El relato es confuso, temeroso, afligido y con presencia de intrusiones visuales en el pensamiento. En este caso, Claudia presenta un estado de la mente no resuelto y atemorizadamente preocupada por eventos traumáticos, esta clasificación es raramente encontrada en muestras de bajo riesgo y se presenta en individuos que han tenido experiencias 
atemorizantes relativas al apego y que estas experiencias son actualmente preocupantes o incluso controlan de manera impredecible los procesos mentales.

En Claudia las experiencias traumáticas la siguen afectando en la actualidad y en la relación con sus hijos. Así, cuando la entrevistadora le pregunta: "¿Y esta experiencia influye algo en el trato con sus hijos?" Responde: "Sí, a veces siento que lo trato a mi hijito mal. Hay veces en que mi hijo no quiere comer y yo no quieres comer ya yaa no hay naadaaa ¿así no? [...] Hay veces que yo lo veo a mi hijo llorar y cuando lo veo llorar ahí sílloro yo con él porque digo yo estoy mal". Se observa cómo al no haber tenido una figura que haya oficiado de cuidado, sostén y contención en su infancia, cuando su hijo llora, Claudia se desborda y se angustia mostrando que ella es la que necesita atención y contención. Esto es característico de los patrones de apego preocupados.

En relación a la conducta sensible de Claudia, se observa un bajo nivel de sensibilidad $(r=.197)$ presentando inadecuadas respuestas ante las señales de su hijo. Además, se encuentra que tiende a interferir en las actividades que realiza el niño y muestra poca preocupación por la apariencia física del mismo. Sus comportamientos más característicos se centran en atender las necesidades físicas de su hijo, por ejemplo, proporcionando refrigerios nutritivos (MBQS, ítem 48) o colocando juguetes a su alcance (MBQS, ítem 90); sin embargo, tiende a desatender el estado emocional de su hijo, ya sea ignorando o demorando la respuesta a las señales de angustia o molestia de su hijo (MBQS, ítem 11), y respondiendo solo cuando estas señales son frecuentes, prolongadas o intensas (MBQS, ítem 7). Esta conducta materna concuerda con lo señalado en la literatura en relación a las madres con estado de la mente no resuelto, manifestándose bajos niveles de disponibilidad emocional y sensibilidad (Moehler et al., 2007). La inhabilidad materna para monitorear su comportamiento interactivo y para responder al estrés de su hijo parecen ser producto de su infancia traumática y su dificultad para resolver e integrar dichas experiencias (Lyon-Ruth \& Jacobvitz, 1999).

Con respecto al hijo de Claudia, éste no ha desarrollado seguridad en el vínculo con su madre $(r=-.322)$, su conducta revela una escasa calidez en las interacciones con su madre y poca búsqueda de su proximidad en situaciones de estrés (AQS, ítem 88). Además, la interacción con otros adultos está disminuida. Se encuentra una conducta de base segura caracterizada por la dificultad para regular sus estados emocionales y encontrar estrategias adecuadas para obtener el cuidado de la madre; así, se molesta ante la ausencia de la madre (AQS, ítem 13), es exigente e impaciente cuando le solicita algo (AQS, ítem 38), comportándose generalmente como si ella no fuera a acceder a su pedido (AQS, ítem 74), por lo que manifiesta sus necesidades a través de la protesta, el llanto o molestia (AQS, ítem 81 y 38). Frente a estas características de la interacción, el estudio de Hsiao et al. (2015) así como el de Negrão, Pereira, Soares y Mesman (2016) señalan que las madres con apego no resuelto y preocupado, como es el caso de Claudia, suelen re-direccionar las interacciones con sus hijos centrando la atención en la propia experiencia emocional y no en la de su hijo/a. Esto generaría que, sobre todo frente a experiencias emocionales negativas como la tristeza, dolor o pena, la madre no pueda organizar la experiencia del niño y no pueda funcionar como una base segura capaz de ayudarlo a resolverlas.

\section{Conclusiones}

En este estudio se describieron las características de las representaciones de apego de dos madres clasificadas con un estado de la mente no resuelto. Si bien en ambos casos se encuentran experiencias no resueltas de situaciones de violencia durante la infancia, éstos presentan diferencias sustanciales en la organización del discurso, lo cual ha llevado a colocar en un caso la sub-clasificación de seguro, a partir de la presencia de elaboración de estas experiencias tempranas y de figuras de sostén alternas presentes en su vida; en el otro caso la subclasificación fue de inseguro, debido a la existencia de preocupación y temor en relación a dichas 
experiencias. Considerando esta sub-clasificación, se ha evidenciado que el caso con un apego No resuelto/Seguro tiene un nivel mayor de sensibilidad materna y de la seguridad del vínculo de su hijo comparado con el caso codificado como No resuelto/Inseguro. Estos resultados son concordantes con la literatura previa.

Los resultados muestran que la clasificación y el análisis detallado de los estados de la mente No resuelto y la sub-clasificación correspondiente, permiten un gran aporte a la comprensión de las experiencias traumáticas al brindar información acerca de los efectos del trauma en el presente y en el propio comportamiento. Esto permite la planificación de una intervención pertinente para cada configuración. Así, en función de los resultados, un tratamiento para Luisa sería trabajar con sus "ángeles" y "fantasmas" presentes a raíz de las experiencias traumáticas vividas, así como sobre sus niveles de ira para fomentar una mayor coherencia en su narrativa y evitar que en algún momento repita o despliegue dicha ira en la relación con sus hijos. Por otro lado, la intervención con Claudia se focalizaría en la comprensión e integración de sus vivencias y en promover un apego seguro con su hijo mejorando su respuesta sensible a las necesidades de su hijo.

Los resultados muestran que el AAI y la observación de la conducta diádica se validan mutuamente, sin embargo, sería pertinente analizar a grupos de madres más amplios, que permitan una mayor sistematización de los resultados. Los resultados serían de gran importancia para el trabajo clínico con madres, especialmente en poblaciones de alto riesgo.

\section{Referencias}

Ainsworth, M., Blehar, M., Waters, E., \& Wall, S. (1978). Patterns of Attachment. Hillsdale, NJ: Erlbaum.

Asociación Peruana de Empresas de Investigación de Mercado [APEIM] (2017). Niveles socioeconómicos 2017. Lima. Recuperado de http://www.apeim.com.pe/wpcontent/themes/apeim/docs/nse/APEIM-NSE-2017.pdf

Bakermans-Kranenburg, M. \& van IJzendoorn, M. (1993). A psychometric study of the Adult Attachment Interview: Reliability and discriminant validity. Developmental Psychology, 29, 870-880.

Bakermans-Kranenburg, M. \& van IJzendoorn, M. (2009). The first 10,000 Adult Attachment Interviews: distributions of adult attachment representations in clinical and non-clinical groups. Attachment y Human Development, 11(3), 223-263.

Ballen, N., Demers, I. \& Bernier, A. (2006). A differential analysis of the subtypes of unresolved state of mind in the adult attachment interview. Journal of Trauma Practice, 5(4), 69-93.

Bárrig-Jó, P., Nóblega, M., Núñez del Prado, J., Conde, G., Carbonell, O., \& Altmann, M. (2016). Attachment Representations and Maternal Sensitivity in Low Socioeconomic Status Mothers. International Journal of Psychological Studies, 8(3), 202-214. doi:10.5539/ijps.v8n3p202

Behrens, K., Haltigan, J. \& Bahm, N. I. G. (2016). Infant attachment, adult attachment, and maternal sensitivity: revisiting the intergenerational transmission gap. Attachment \& human development, 18(4), 337-353.

Berthelot, N., Ensink, K., Bernazzani, O., Normandin, L., Luyten, P., \& Fonagy, P. (2015). Intergenerational transmission of attachment in abused and neglected mothers: The role of trauma-specific reflective functioning. Infant Mental Health Journal, 36(2), 200-212.

Bowlby, J. (1969/1982). Attachment and Loss: Vol. 1. Attachment. New York: Basic Books.

Crugnola, C. R., Gazzotti, S., Spinelli, M., Ierardi, E., Caprin, C., \& Albizzati, A. (2013). Maternal attachment influences mother-infant styles of regulation and play with objects at nine months. Attachment \& human development, 15(2), 107-131.

Fonagy, P., Steele, M., Moran, G., Steele, H. \& Higgit, A. (1993). Measuring the ghosts in the nursery: An empirical study of the relation between parents' mental representations of childhood experiences and their infants' security of attachment. Journal of the American Psychoanalytic Association, 41(4), 957-989. 
Fraley, R., \& Shaver, P. (2016). Attachment, loss, and grief: Bowlby's views, new developments, and current controversies En J. Cassidy \& P. R. Shaver (Eds.), Handbook of attachment: Theory, research, and clinical applications ( $3^{\text {rd }}$ ed.), (pp. 40-62). New York: Guilford Press.

George, C., Kaplan, N., \& Main, M. (1985). The Adult Attachment Interview. Unpublished manuscript, University of California at Berkeley.

Goldberg, S., Benoit, D., Blokland, K. \& Madigan, S. (2003). Atypical maternal behavior, maternal representations, and infant disorganized attachment. Development and Psychopathology, 15(2), 239-257.

Grice, P. (1975). Logic and conversation. En P. Cole \& J.L. Moran (Eds.), Syntax and Semantics III: Speech Acts (pp. 41-58). New York, Academic Press.

Hesse, E. (2016). The Adult Attachment Interview: Protocol, method of analysis, and empirical studies: 1985-2015. En J. Cassidy \& P. R. Shaver (Eds.), Handbook of attachment: Theory, research, and clinical applications ( $3^{\text {rd }}$ ed.), (pp. 553-597). New York: Guilford Press.

Hesse, E. \& Main, M. (2000). Disorganized infant, child and adult attachment: Collapse in behavioral and attentional strategies. Journal of the American Psychoanalytic Association, 48(4), 1097-1127.

Hsiao, C., Koren-Karie, N., Bailey, H., \& Moran, G. (2015). It takes two to talk: Longitudinal associations among infant-mother attachment, maternal attachment representations, and motherchild emotion dialogues. Attachment \& human development, 17(1), 43-64.

Instituto Nacional de Estadística e Informática [INEI]. (2016). Encuesta Demográfica y de Salud Familiar. Lima: Instituto Nacional de Estadística e Informática.

Lieberman, A., Padrón, E., Van Horn, P. \& Harris, W. (2005). Angels in the nursery: The intergenerational transmission of benevolent parental influences. Infant Mental Health Journal, 26(6), 504-520.

Lyons-Ruth, K. \& Jacobvitz, D. (1999). Attachment disorganization: Unresolved loss, relationship violence, and lapses in behavioral and attentional strategies. En J. Cassidy \& P. Shaver (Eds.), Handbook of attachment: Theory, research, and clinical applications (pp. 520-554). New York: Guilford Press.

Madigan, S., Bakermans-Kranenburg, M., van IJzendoorn, M., Moran, G., Pederson, D. \& Benoit, D. (2006). Unresolved states of mind, anomalous parental behavior and disorganized attachment: A review and meta-analysis of a transmission gap. Attachment and Human Development, 8(2), 89111.

Madigan, S., Hawkins, E., Plamondon, A., Moran, G., \& Benoit, D. (2015). Maternal representations and infant attachment: An examination of the prototype hypothesis. Infant mental health journal, $36(5), 459-468$.

Main, M. \& Hesse, E. (1990). Parents' unresolved traumatic experiences are related to infant disorganized attachment status: Is frightened and/or frightening parental behavior the linking mechanism? En M.T. Greenberg, D. Cicchetti, \& E. Cummings (Eds.), Attachment in the preschool years: Theory, research, and intervention (pp. 161-182). Chicago: University of Chicago Press.

Main, M. \& Goldwyn, R. (2004). Sistema de Calificación y Clasificación de la Entrevista de Apego Adulto. Manuscrito no publicado, versión 7.1. (Gojman de Millán, trad.). México: S. Universidad Nacional Autónoma de México. (Obra original publicada en 2002).

Main, M., Kaplan, N., \& Cassidy, J. (1985). Security in infancy, childhood, and adulthood: A move to the level of representation. Monographs of the Society for Research in Child Development, 50(1-2, Serial No. 209), 66-104.

Moehler, E., Biringen, Z., \& Poustka, L. (2007). Emotional availability in a sample of mothers with a history of abuse. American Journal of Orthopsychiatry, 77(4), 624.

Negrão, M., Pereira, M., Soares, I., \& Mesman, J. (2016). Maternal attachment representations in relation to emotional availability and discipline behaviour. European Journal of Developmental Psychology, 13(1), 121-137.

Nóblega, M., Bárrig, P., Conde, L. G., Nuñez del Prado, J., Carbonell, O. A., Gonzalez, E.... \& Bauer, M. (2016). Cuidado materno y seguridad del apego antes del primer año de vida. Universitas Psychologica, 15(1), 245-260. doi: 10.11144/Javeriana.upsy15-1.cmsa.

Pederson, D. \& Moran, G. (1995). Appendix B. Maternal Behavior Q-set. En E. Waters, B. E. Vaughn, G. Posada, \& K. Kondo-Ikemura (Eds.) Caregiving, cultural, and cognitive perspectives on secure- 
base behavior and working models: New Growing Points of Attachment Theory and Research. Monographs of the Society for Research in Child Development 60(2-3), 247-254.

Posada, G., Jacobs, A., Richmond, M., Carbonell, O., Alzate, G., Bustamante, M., \& Quiceno, J. (2002). Maternal caregiving and infant security in two cultures. Developmental Psychology, 38(1), 67-78. doi:10.1037/0012-1649.38.1.67

Reijman, S., Alink, L., Compier-De Block, L., Werner, C., Maras, A., Rijnberk, C., ... \& BakermansKranenburg, M. (2016). Attachment representations and autonomic regulation in maltreating and nonmaltreating mothers. Development and psychopathology, 1-13.

Saunders, R., Jacobvitz, D., Zaccagnino, M., Beverung, L., \& Hazen, N. (2011). Pathways to earnedsecurity: The role of alternative support figures. Attachment \& human development, 13(4), 403420.

Schuengel, C., Bakermans-Kranenburg, M. \& van IJzendoorn, M. (1999). Attachment and loss: Frightening maternal behavior linking unresolved loss and disorganized infant. Journal of Consulting and Clinical Psychology, 67(1), 54-63.

van IJzendoorn, M. (1995). Adult Attachment Representations, Parental Responsiveness and Infant Attachment: A Meta-Analysis on the Predictive Validity of the Adult Attachment Interview. Psychological Bulletin, 117(3), 387- 403.

van IJzendoorn, M. \& Bakermans-Kranenburg, M. (1996). Attachment representations in mothers, fathers, adolescents, and clinical groups: A meta-analytic search for normative data. Journal of Consulting and Clinical Psychology, 64(1), 8-21.

Verhage, M., Schuengel, C., Madigan, S., Fearon, R., Oosterman, M., Cassibba, R., BakermansKranenburg, M., \& van IJzendoorn, M. (2016). Narrowing the transmission gap: A synthesis of three decades of research on intergenerational transmission of attachment. Psychological Bulletin, 142(4), 337-366.

Waters, E. (1995). The Attachment Q-Set (Version 3). En E. Waters, B. Vaughn, G. Posada \& K. KondoIkemura (Eds.), Caregiving, cultural, and cognitive perspectives on secure-base behavior and working models: new growing points of attachment theory and research. Monographs of the Society for Research in Child Development, 60(2-3), 234-246.

Zaccagnino, M., Cussino, M., Saunders, R., Jacobvitz, D., \& Veglia, F. (2014). Alternative caregiving figures and their role on adult attachment representations. Clinical psychology \& psychotherapy, $21(3), 276-287$.

Contribución de los autores: a) Concepción y diseño del trabajo; b) Adquisición de datos; c) Análisis e interpretación de datos; d) Redacción del manuscrito; e) revisión crítica del manuscrito.

M.N. ha contribuido en a,b,c,d,e; L.S. en c,d,e ; F.M. en c,d,e; P.B.J. en a,b,c; J.N.P. en a,b,c; G.C. en a,b,c.

Editora científica responsable: Dra. Cecilia Cracco 